\title{
Bericht von der FSJ-Jahrestagung am 3. und 4. Dezember 2012 in Berlin
}

\author{
Dörte Lüdeking \\ DRK-Freiwilligendienste im DRK-Generalsekretariat $\mid$ Referentin \\ luedekid@drk.de \\ Dr. Jaana Eichhorn \\ Freiwilligendienste im Sport bei der Deutschen Sportjugend | Bundestutorin \\ eichhorn@dsj.de
}

Am 3. und 4. Dezember 2012 fand die vom Bundesministerium für Familie, Senioren, Frauen und Jugend (BMFSFJ) veranstaltete und in Kooperation mit dem Bundesarbeitskreis Freiwilliges Soziales Jahr (BAK FSJ) vorbereitete FSJ-Jahrestagung in Berlin statt. Die Tagung, die unter dem Titel „Veränderung gestalten - Qualität sichern“ stand, behandelte Themen wie qualitative Grundlagen, Mindeststandards und Qualitätsmanagement im FSJ. Die Veranstaltung war mit über 120 TeilnehmerInnen sehr gut besucht und bildet einen wertvollen Beitrag im Diskussionsprozess rund um eine mögliche gemeinsame Qualitätsvereinbarung in den Jugendfreiwilligendiensten. Sowohl das BMFSFJ als auch der BAK FSJ hoben hervor, dass eine Beschäftigung mit Qualitätsstandards einen bewusst gesetzten Kontrapunkt zu den Veränderungen in den Förderbedingungen und den damit verknüpften Herausforderungen bilden soll. Ziel der Veranstaltung war es, den Austausch zwischen den Akteuren im FSJ zu fördern und insbesondere den Trägern Impulse für die eigene Arbeit zu vermitteln. Zugleich sollte darüber diskutiert werden, welche Aspekte und Elemente für zukünftige Qualitätsvereinbarungen für alle FSJ-Anbieter sinnvoll und notwendig erscheinen.

Ausgangspunkt der Tagung war das im Wandel begriffene Qualitätsverständnis im Freiwilligen Sozialen Jahr: Träger und Einsatzstellen stellen seit fast fünfzig Jahren gemeinsam mit ihren Bundestutoraten, Bund und Ländern sicher, dass den jungen Freiwilligen ein hochwertiges Angebot unterbreitet wird. Nun haben sich die Rahmenbedingungen der Jugendfreiwilligendienste, und damit auch des FSJ, verändert. Das FSJ ist aus der Nische in die Mitte der Gesellschaft gerückt und wird dort deutlich wahrgenommen: Wesentlich mehr junge Freiwillige als je zuvor leisten derzeit ein FSJ. Dies 
zeigt einerseits, dass Jugendliche und junge Erwachsene offen sind für ein freiwilliges Engagement für die Gesellschaft. Andererseits besteht aber bei ihnen gerade in Anbetracht des jüngeren Alters beim Schulabschluss oft ein hoher Bedarf, sich zu orientieren, die Facetten der eigenen Persönlichkeit nach dem Schulbesuch weiter auszuleuchten und mit Blick auf eine spätere berufliche Tätigkeit die eigenen Stärken oder Schwächen im praktischen Einsatz zu erforschen. Durch den Ausbau und die veränderte Struktur seiner Förderung trägt der Bund dazu bei, dass die Träger die steigende Zahl an Freiwilligen finanziell bewältigen können. Da Qualität nicht statisch ist, muss sich das FSJ, auch mit Blick auf den Mitteleinsatz, immer wieder der Qualitätsfrage stellen und sich - besonders auch unter den veränderten Bedingungen - qualitativ weiterentwickeln.

In zwei Grundsatzreferaten befassten sich zunächst Prof. Dr. Bernd Overwien von der Universität Kassel sowie Prof. Dr. Joachim Merchel von der Fachhochschule Münster mit Chancen, Risiken und Nebenwirkungen des Qualitätsdiskurses im Bildungs- bzw. Jugendhilfebereich. Insbesondere Prof. Merchel, der zum Qualitätsmanagement in der sozialen Arbeit breit publiziert hat, machte deutlich, dass Qualitätsvereinbarungen kein Selbstzweck sein dürfen. Ziel müsse es sein, sich auf klar messbare Zielsetzungen zu einigen und die Zielerreichung dokumentieren zu können, ohne zusätzliche Bürokratie zu verursachen. Viele der TrägervertreterInnen, die sich gemeinsam mit ihren Zentralstellen in unterschiedlichen Qualitätsentwicklungsprozessen befinden, unterstützten diese Auffassung mit eigenen Erfahrungen.

In einer Podiumsdiskussion, die von Ana-Maria Stuth (Quifd - Qualität in Freiwilligendiensten) sachkundig geleitet wurde, diskutierten die Grundsatzreferenten gemeinsam mit Dr. Jens Kreuter, dem Leiter des Arbeitsstabes Freiwilligendienste im BMFSFJ, Dr. Andreas Frank, Referatsleiter im Bayerischen Sozialministerium sowie Dörte Lüdeking, Sprecherin des BAK FSJ. Im Zentrum stand die Frage nach einer Qualitätsvereinbarung zwischen Trägern bzw. ihren Zentralstellen sowie dem Bund. Deutlich wurde, dass die Qualität in den Jugendfreiwilligendiensten so hoch ist, dass es nur darum gehen kann, einzelne „schwarze Schafe“ herauszufiltern, nicht aber, Unterschiedlichkeiten um jeden Preis zu beseitigen. Der BAK FSJ erklärte, dass eine Qualitätsvereinbarung mit einer Fördervereinbarung verbunden werden müsste, um einen Mehrwert für alle Beteiligten zu sichern. Dabei wurde 
deutlich, dass das FSJ bereits für eine langjährig bewährte Qualität steht. Die Bundestutorate, Träger und Einsatzstellen stellen im Rahmen ihrer täglichen Arbeit gemeinsam mit dem Bund und den Ländern sicher, dass den Freiwilligen ein sinnvolles, ihren Bedürfnissen angepasstes Angebot gemacht wird.

Wichtige Impulse gingen von den verschiedenen Arbeitsgruppen aus. Arbeitsgruppe 1, moderiert von Ute Giesecke-Tapp von den evangelischen Freiwilligendiensten, befasste sich mit der Bestandsaufnahme aktueller FSJMindeststandards. $\mathrm{Zu}$ konstatieren ist, dass es bereits eine Vielzahl von Standards im FSJ gibt: Dies sind zum einen spezifische Standards einzelner Trägergruppen für ihren jeweiligen Bereich, zum anderen trägerübergreifende Vereinbarungen. Diskutiert wurde, welche Gemeinsamkeiten in den bestehenden Standards zu finden sind und worin sie sich unterscheiden. Kontrovers wurde untersucht, wie flexibel beziehungsweise wie konkret Standards gefasst werden sollen und welche Bereiche betroffen sein sollen.

In der zweiten Arbeitsgruppe, die unter der Leitung von Kerstin Hübner (Bundesvereinigung Kinder- und Jugendbildung) stand, wurde die Rolle von Evaluation und Wirksamkeit im Qualitätsdiskurs unter die Lupe genommen. Systematische Evaluationsprozesse tragen dazu bei, neben der Ergebnisqualität die Struktur- und Prozessqualität zu überprüfen. Ein wichtiger Qualitätsaspekt des FSJ ist aber auch dessen Wirkung. Die Arbeitsgruppe diskutierte, welche Evaluationsinstrumente (z. B. Selbstevaluationskonzepte, Seminarauswertungen, Befragung von Freiwilligen, Ehemaligen und Einsatzstellen) in den einzelnen Trägerverbünden genutzt werden, wozu sie sich eignen und welche Erkenntnisse bereits ermittelt wurden. Kritisch betrachtete die Arbeitsgruppe die Frage, welchen Einfluss Evaluationsergebnisse und Wirkungsstudien auf die Weiterentwicklung von Qualität im FSJ haben können und sollen.

Die dritte Arbeitsgruppe befasste sich unter der Leitung von Hartmut Brombach (Internationaler Bund) mit der Partizipation von Freiwilligen und Einsatzstellen an der Qualitätsdiskussion. Ziel ist es, das FSJ als ein Angebot zu gestalten, das den Bedürfnissen und Interessen der jungen Freiwilligen entspricht und diese in Einklang mit den Bedarfen und Möglichkeiten von Einsatzstellen bringt. Insofern ist es notwendig, Freiwillige und Einsatzstellen an der Qualitätsentwicklung im FSJ aktiv zu beteiligen. Zudem ist die Partizipation von Jugendlichen, die aktive Mitgestaltung von Freiwilligen an „ihrem“ FSJ, ein wichtiges Qualitätsmerkmal der Jugendhilfe und des Bil- 
dungsbereichs und entsprechend gesetzlich verankert. Wie die Diskussion ergab, nutzen die Träger eine Vielfalt an Partizipationsmöglichkeiten - für Einsatzstellen z. B. Besuche und Treffen, Befragungen und Unterstützungsangebote; für Freiwillige z. B. das Sprechersystem und Arbeitsgruppen, Seminarvorbereitungen und Mentoringkonzepte. Gemeinsam wurde überlegt, welche Rolle diese Möglichkeiten im Rahmen der Qualitätsdiskussion spielen und wie sie gestärkt werden können.

Die vierte Arbeitsgruppe, moderiert von Axel-Kurt Schubert (Bundesamt für Familie und zivilgesellschaftliche Aufgaben) nahm veränderte Zielgruppen und ihren Einfluss auf die Qualitätsentwicklung in den Blick. Die Gruppe der Teilnehmenden des FSJ hat sich in den letzten Jahren verändert, wozu der Ausbau der Jugendfreiwilligendienste und der damit verbundene starke Anstieg an interessierten Freiwilligen aus allen Gesellschafts- und Bevölkerungsschichten entscheidend beigetragen hat. Auch die Zahl der Freiwilligen mit besonderem Förderbedarf hat sich dadurch erhöht; so entscheiden sich auch immer mehr sehr junge Freiwillige, aber auch solche mit psychosozialen Erkrankungen für ein FSJ. Diese Zielgruppen, aber z. B. auch Freiwillige mit Studienabschluss, stellen zunehmend besondere Anforderungen an die Begleitung durch Einsatzstellen und Träger. Die AG diskutierte, in welchem Umfang das FSJ diese neuen Anforderungen erfüllen kann und will und wie es gelingt, sich de facto auf die Bedürfnisse einer neuen Klientel einzustellen. Dabei wurden auch die Grenzen einer Öffnung des FSJ wiederholt deutlich.

Die Arbeitsgruppe 5 befasste sich unter der Leitung von Tina Stampfl (Bundesverband Arbeiterwohlfahrt) mit „Lernzielen“ und Kompetenzdokumentation als Instrumenten der Qualitätsentwicklung. Seit mehreren Jahren bewegt Träger, Einsatzstellen und Freiwillige im FSJ - u. a. aufgrund der Gesetzesnovellierung 2008 - wie die Lernziele eines FSJ bestimmt und beschrieben werden können und wie sich ihre Erreichung belegen lässt. Anknüpfend an zahlreiche Debatten im Bildungsbereich spielt besonders der Kompetenzbegriff eine zunehmend wichtige Rolle. Verschiedene Träger haben Verfahren zur Kompetenzdokumentation entwickelt und erprobt. Diskutiert wurde, ob sich diese Verfahren als für das FSJ tauglich erwiesen haben und ob sie die Qualitätsentwicklung im FSJ unterstützen. Auch das Spannungsfeld zwischen Lernzielvereinbarungen oder Kompetenzbilanzierungen einerseits und 
dem informellen und non-formalen Bildungsansatz des FSJ andererseits wurde thematisiert.

Auch wenn es nicht zu allen Fragen abschließende Antworten geben konnte, schätzten die TeilnehmerInnen, dass statt verwaltungs- und fördertechnischer Fragen endlich wieder das FSJ mit seinen Inhalten im Mittelpunkt stand. Der Austausch fand auf fachlich hohem Niveau statt und konnte so zur inhaltlichen Weiterentwicklung des Themas beitragen. Viele TeilnehmerInnen konnten neue Impulse für die eigene Arbeit von der gelungenen Tagung auf die Heimreise mitnehmen. Charlotte Hüllen vom BMFSFJ betonte abschließend, dass die inhaltliche Ausgestaltung des Feldes die Kernaufgabe der Freiwilligendienstakteuere sei. Das Ministerium werde die Tagungsergebnisse auswerten und zur Weiterentwicklung nutzen; hierzu würden weitere Gespräche mit Zentralstellen und Trägern geführt. Eine Dokumentation der Tagung ist angekündigt.

\title{
Internationale Freiwilligendienste: Konzept einer neuen Freiwilligenvertretung für alle
}

\author{
Lilia Afroune, Michel Arnd, Arne Bonhage, Rabea Brandt, Bastian \\ Göckler, Joschka Köck, Matthias Scharpenberg, Tabea Schroer \& \\ Marian Peters...
}

... haben sich als offenes Team auf dem von grenzenlos e.V. - Vereinigung internationaler Freiwilliger initiierten Politischen Treffen und der undjetzt?!-Konferenz zusammengefunden und das folgende Konzept mit dem Ziel einer unabhängigen Freiwilligenvertretung entwickelt. pfif@freiwilligenvertretung.de

\section{Einleitung}

Die Freiwilligendienstlandschaft hat sich mit der Einführung geregelter Dienste wie des entwicklungspolitischen Freiwilligendienstes weltwärts und des Freiwilligendienstes des Auswärtigen Amtes kulturweit, wie auch mit der Etablierung des Internationalen Jugendfreiwilligendienstes (IJFD) entscheidend verändert und $\mathrm{zu}$ einem sprunghaften Anstieg an Entsendungen 